\title{
Evaluating UX using a Metric Model: the Case of a Handheld Computing System in South Africa
}

\author{
Guy Toko, Ernest Mnkandla
}

\begin{abstract}
For most experienced graphical user interface (GUI) and interactive designers, succeeding in acquiring, maintaining, and guiding potential high-level user engagement and user experience $(\mathrm{UX})$ at the first attempt is often a dream. It often takes many low- and high-fidelity prototypes to obtain the desired solution, if not abandoning the entire activity altogether, due to mounting pressure and disappointment after failing to satisfy user needs while maintaining industry standards and design principles. The challenges are often due to the absence of known, agreed-upon evaluation mechanisms that are known and acceptable to interactive designers. The aim of this paper is to introduce an instrument that can be used to measure and evaluate $U X$, which can be used at any time during the design process and limits the pressure interactive designers too often experience.
\end{abstract}

Keywords: Evaluating-UX, UX-metric-model, handheldcomputing

\section{INTRODUCTION}

The computing systems present everywhere in our lives are here to stay. Our dependence on them is constantly growing and our ability to break free is diminishing day after day (Turban et al. 2008:41). Our introduction to computing systems is a thing of the past, yet we are constantly being reintroduced to them as if we are coming into contact with them for the very first time $(\mathrm{Oz} \&$ Jones 2008:40). Their presence is so obvious and invasive that we are constantly keeping track of them and observing the next trend to ensure that we are the first to discover new products (Shely, Cashman \& Walker 2015:51). User needs are constantly changing due to personal aspirations and circumstances, and so are the new features that computing system designers need to introduce to the next development in order to please us and satisfy those needs (Lee \& Kim 2015:212).

The constant innovative nature of computing systems makes them an ideal technological instrument that can be used to positively impact society and, most importantly, improve the living standard of those who still have limited contact with them on a daily basis (Lee \& Kim 2015:200). They have impacted almost all aspects of our lives; from the way humans sleep and wake up, travel, eat, and communicate, to the way we interact with everyone and everything around us on a daily basis and beyond (Reiss 2012:30). The impact of computing systems and overall system usability is so important that numerous scientific studies have been conducted with the aimof investigating their impact, their progression, and how to foresee their evolution and possibly control them in the short, medium, and long term (Prietch \& Filgueiras 2015:20). Since humans come into direct contact with these tools, one needs to

Revised Manuscript Received on October31, 2019

* Correspondence Author

Guy Toko*, Applies Information Systems Department of the University of Johannesbur, Johannesburg, South Africa. Email: gtoko@uj.ac.za

Ernest Mnkandla, Department of Computer Science, School of Computing, University of South Africa, Johannesburg, South Africa. Email: emnkanla@unisa.ac.za also investigate the relationship between these technological tools and humans, exploring important aspects such as UX, which is in most cases the responsibility of interactive designers. The question interactive designers too often ask themselves during the design process is how users will react when exposed to the new systems. It is no secret that computing innovation is driven by individualism and fear of numerous copyright laws and patents; hence, no system developer wants to be caught imitating their competitors, and users at the end of the development chain need to be the adjudicators, yet there is no adjudication instrument (Turban et al. 2008:41)

\section{BACKGROUND}

This study explores UX with the aim of developing a UX assessment tool that can be used to evaluate the level of user interaction with and adaptation to a new computing system. In this study, computer users are all seen as potential users, with technological needs and aspirations that need to be met by system developers. The study includes first-time users, as they may not have been in contact with any form of computing system before. It is also important to note that this study is confined to the South African context.

The computing system instrument used in this study is a handheld computing system in the form of a tablet as it represents one of the most commonly used computing systems and is also widely accessible, given the current market cost and availability.

The indirect question that can also be asked here is when and how an interactive designer may evaluate the level of usability, user-friendliness, and inherent UX of a potential computing system before its public release.

For some people, a handheld computing system comes as a supplement to their desktop or laptop computer, or is one of their multiple personal computing systems; but for others, perhaps the majority of South Africa's aspiring computer users, it may be the only computing system they will ever own (Gilward, Moyo \& Stork 2010:20) and most likely the only computing device they can afford that allows them to stay in touch with the rest of the world (Toko \& Mnkandla 2011:151).

There is no doubt that high levels of UX create better and continuous users. For a country like South Africa, that will be ideal, since it would be in line with the government's aim of creating a digitalised society. Also, potential users with low levels of UX may slip through the cracks of entering the digital space if the GUI they encounter for the first time is not suitable or was poorly designed.

Unlike most developed nations, the number of people who still want to learn how to operate a computing system in our society is immense. 
These untapped user communities can be young or very old, and some of them may never have had an opportunity to access or use a computing device in their lives, for reasons such as their geographical location, advanced age, poor school grades, or low literacy level (Vatavu, Anthony \& Brown 2015:1).

The history of South Africa exposed some sections of the population to high levels of various vulnerabilities, which resulted from many negative and depriving types of human segregation, and further contributed to two distinct segments of the country's population (Adebajo 2016:14).

The authors are of the view that a study of this magnitude is important as it highlights the academic research contribution to the improvement of the quality of information and communications technology (ICT) within our communities. Academia has always been at the forefront of social issues, searching for and providing answers to unexplained and unforeseen phenomena, guiding decision makers regarding the impact of some of their policies, and discovering new ICT tools, among others (Delanty 2005:13).

\section{LITERATURE REVIEW}

\section{A. Understanding the role of $U X$}

$\mathrm{UX}$ is slowly becoming an independent subfield within the human-computer interaction (HCI) spectrum. Many new $\mathrm{HCI}$ or interactive researchers are becoming interested in this field solely based on the fact that it is becoming an increasingly important issue within the usability field (Yu \& Kim 2015:550). It contains some very intriguing aspects of interactive system development or, more specifically, GUI design characteristics.

UX is the central piece of any form of interaction development regarding computer systems (Yu \& Kim 2015:550). One will not be wrong in stating that UX encompasses how any user deals with a device, or how any user feels while interacting with a device ( $\mathrm{Yu} \& \mathrm{Kim}$ 2015:550). UX is described as a binding concept that reflects the services offered, the actual device, and the developing team; all of which later create user anticipation and execution of the ultimate prototype or product (Yu \& Kim 2015:550).

It may be naive to blindly state that UX is simply about good industrial design, multi-touch interactions, or a fancy interface, rather than a perception that transcends the physical material, and more about creating or inventing an emotional experience and contact through the handheld computing device (Yu \& Kim 2015:550). This includes any device, regardless of the type, design accuracy, degree of interactivity, and robustness of its internal memory, processing speed, and cost of acquisition ( $\mathrm{Ni}, \mathrm{Al}$ Mahmud \& Keyson 2015:546).

The lasting emotional experience of the user is a sort of ultimate "baptism" the user has when interacting with a handheld computing system, such as a tablet, for the very first time, which may be a profound experience for a user $(\mathrm{Ni}$ et al. 2015:546). It creates a UX in the true sense of the word.

UX involves a user's deep emotions about and devotion to the computing product, and highlights the experiential, affection, connotation, and value factors of the HCI aspect of the device ( $\mathrm{Ni}$ et al. 2015:546). It involves the sensory effect a user may feel after touching or tapping a particular link or pressing on a particular task, for example, how itfeels after clicking a button, tapping on a particular icon, and how text is displayed after keys are pressed. Nevertheless, it should also be stressed that, during the development of computing systems, it is almost impossible to design "any UX", but one may design "for UX" (Ni et al. 2015:546). This simply means that even the best designer may not design a sensual experience but rather include some particular design features that may have a sensual effect on the user (Day, Buchanan\&Makri 2015:578).

At times, the UX may be subjective in its natural sense. It is more about individual views and imagination with regard to the system versus the known experiment, and at times designers immerse themselves in the user's natural environment to emulate their line of thinking. It may also be dynamic, as it is constantly modified over time due to changing circumstances and new publications on the subject (Day et al. 2015:578).

It is impossible for a designer to sit for days on end to design any UX. No designer can create UX by simply documenting it, but the ideal design may come as a designer applies it, in other words, by doing it (Day et al. 2015:578). In any true and acceptable GUI design, there is a need to design a product that offers the best UX for any potential user. The magnificence of any product first comes from its external physical appearance and then from how the user feels after touching it for the very first time (Leo de Oliveira, Miletto \& Flores 2015:590). It is an empirical goal to develop a product that does what it is supposed to do and makes sense to the user, a device that has all the best and innovative features one may wish for. However, this may no longer be sufficient because the designer also needs to develop devices that are understandable, usable, exciting, user friendly, and fun for its user (Leo de Oliveira et al. 2015:590).

McCarty and Wright (2010:212) wrote the following:

There are four core threads that make up our holistic experience: sensual, emotional compositional, and spatiotemporal:

The sensual thread: This is concerned with our sensory engagement with a situation and is to the visceral level of Norman's model. It can be equated with the level of absorption people have with various technology devices and applications. Most notable being computers games, smartphones, and chat rooms, where users can be highly absorbed in their interactions at a sensory level. These can involve thrill, fear, pain, and comfort.

The emotional thread: Common examples of emotion that spring to mind are sorrow, anger, joy, and happiness. In addition, the framework points out how the emotions are intertwined with the situation in which they arise. Example: a person becoming angry with a computer because it does not work properly. Emotions involve making judgements of value. For example, when purchasing a new cell phone, people may be drawn to the ones that are most cool-looking but be in an emotional turmoil because they are the most expensive. They cannot really afford them but they would really like to have them.

The compositional thread: This is concerned with the narrative part of an experience as it unfolds and the way a person makes sense of it. Example: when shopping online, the option laid out to people can lead them in a coherent way to making a desire purchase or they can lead to frustrating experiences resulting in no purchase being made.

When in this situation, people ask themselves questions such as: What is this about? Where am I? What happened? What is going to happen next? What would happen if...? The compositional thread is the internal thinking we do during our experiences. 
The spatio-temporal thread: This refers to the space and time in which our experiences take place and their effect upon those experiences. There are many ways of thinking about space and time and their relationship with one another. Example: we talk of time speeding up, standing still, and slowing down, while we talk of space in terms of public and personal places, and needing one's own space.

The UX is extremely important in comprehending how user assessment must be conducted. The current absence of reliable forms of evaluation is paramount to the total absence of control mechanisms in a society that needs to evaluate almost all types of activity. A well-built UX metric can address the issue of subjectivity that is observed regarding user computer skills and the adoption thereof.

\section{1) Handheld user challenges}

Regarding handheld computing devices, users must first deal with the challenges of having to physically control the device with one hand and having the other hand operate it while moving around or standing still (Lee, Lee \& Kim 2015:607). Whether the user is left- or right-handed, the challenge is the same; only one hand is freely available (Lee \& Kim 2015:203)

The other challenge that any user faces is the actual interaction; whether using the stylus or the finger to tap, the user needs to think very carefully about how the system might react. At times, the link may not be as visible as the user may expect, or their adult fingers are too big for the contact point where one needs to tap, which results in selecting and accessing undesired targets (Liang et al. 2011:607).

Navigation is one of the most difficult aspects to control for most adult first-time users. Some may have had basic traditional computing experience and may want to navigate in the same way as when using their desktop computing system, but they will still be confronted with unexpected mobility problems (Marquardt 2015:644). Controlling tabs for some may be as hard as any other navigation problem; they may want to return to the main screen without using an external button (Marquardt 2015:644).

The most common hurdle for handheld computing system users is the emotional aspect during the interaction. Most are fearful of the device, while others, although they are current cellphone users, cannot clearly see the link between the tablet system and their cellphone(Marquuardt 2015:644) and tend to move away from their existing cell phone's usability knowledge and attempt to use the tablet as if it was a completely new device (Marquardt 2015:644).

Figure 1 displays three ways in which tablets can be handled and manipulated. For most ICT experts, this is why using handheld devices is so hard, as users must not only hold and control the device but must also interact with it. One should also know if the user is left- or right-handled in order to use the system because this has to do with system orientation and control.

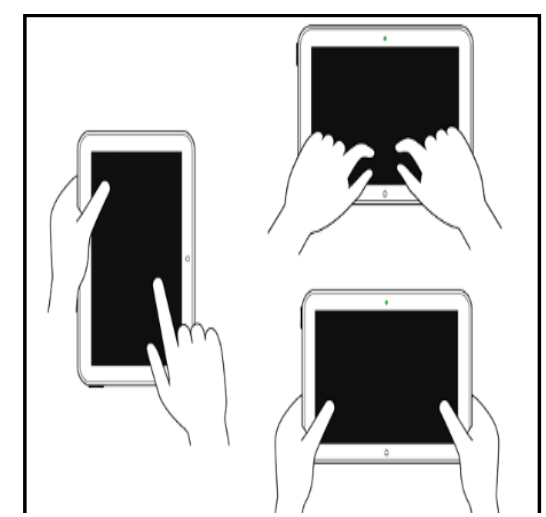

Fig 1: Types of interactions with tablets

Source: Duncan (2015)

\section{RESEARCH VARIABLES}

1) Experiments

When conducting our experiment, we used an algorithm built by ourselves and implemented using a Microsoft Excel spreadsheet in order to develop our metric. We made use of common and existing mathematical formulae and visualised and measured the patent migration that constitutes the various usability orientations. To fully comprehend all the key performance indicators as discussed below, one needs to keep an eye on the mathematical table that contains the description and formulation. The following are just some of the user characteristics we attempted to assess.

2) Movement time (MT)

MT is the time it takes a particular participant to move from one point to another after instruction. In order to do this, certain variables are needed, such as the movement distance from the starting position to the target; the target width; some of the empirically determined constants, such as a and $\underline{b}$; as well as a constant such as $\underline{\mathrm{c}}$, which is 0 or 0.5 . The application of Fitts' model plays an important role in this section.

\section{3) Completion time (CT)}

$\mathrm{CT}$ is the time it takes to complete a task. A task here can be a single unit of action; for example, from A to B. It can also be the CT of a set of tasks per unit, such as the completion of five tasks that are all part of the same unit, for example, the first eight tasks of the same position. Position refers to a set of activities performed with a specific device. There are various CTs, which take into consideration the lapse time, which refers to the time a participant takes to start engaging with a task when told to do so. The users' starting and ending times were known.

4) Activities effectiveness (AE)

$\mathrm{AE}$ is one of the most important factors that needed to be assessed in this study. It refers to the effectiveness of a user's action during the assessment. To determine this, the number of tasks completed successfully is divided by the total number of tasks undertaken, which is then multiplied by $100 \%$ to determine its effectiveness.

5) Success rate $(S R)$

This formula determines the SR of all the tasks and activities performed during the experiment. In order to do this, the performance index must be taken into account. It is important to note that there are various sets of SRs: the individual SR, as well as the position, different participants across the same task, as well as horizontal and vertical comparison. 
Evaluating UX using a Metric Model: the Case of a Handheld Computing System in South Africa

$M T=a+b . I D=a+b \cdot \log _{2}\left(1+\frac{D}{W}\right)$
Overall efficiency $=\frac{\sum_{j=1}^{R} \sum_{i=1}^{N} n_{i j} t_{i j}}{\sum_{j=1}^{R} \sum_{i=1}^{N} t_{i j}} \times 100$
Effectiveness
Numberof tasks completed successfully
Totalnumberof tasksundertaken
$v_{t s}=\frac{D}{M T}$
IP $=\frac{\text { ID }}{\text { MT }}$

6) Task speed (TS)

The different types of speed of the participants were assessed, which included single participant speed, vertical and horizontal speed per task, position, and overall speed. To calculate this, the starting time and the constant are needed. This is basically the speed that is required to complete a task that would be calculated by computing the time it takes to complete a single task and accumulated overall task time. The formula is presented in Table 1 .

\section{7) Activity speed (AS)}

AS is the time that is needed to complete a single action. Like TS, AS is calculated by paying attention to the action that is needed to accomplish a single movement, such as moving from point $\mathrm{A}$ to point $\mathrm{B}$, and it is measured in centimetres per second. It reflects the time it took a participant to move from one position to another and, like TS, is vertically and horizontally applied and measured.

\section{8) User satisfaction (US)}

Here we assessed the level of US. This was done by exploring the participants' efficiency performance versus the maximum percentage allocation for the task. By calculating the US in this manner, the researchers intended to assess the ability of adult first-time users versus system correlation, which can later determine the overall system satisfaction and adaptability.

\section{9) Reaction time (RT)}

RT is the time it takes a user to psychologically comprehend the activity to be completed or the time a user takes before completing the requested activity. It is one of the fundamental principles that have an impact on the user. It not only involves the ability to complete a task but also the comprehension time that is needed for anyone to begin interacting with the task. RT is sometimes synonymous with real starting time, when the user actually engages with the action that is needed or demanded.

10) Completion rate per task and activity (CRTA)

CRTA is the user's ability to complete a task and activity within the set spectrum and conditions. When an activity is requested, it may not always be completed as per the requirement, and the user cannot reach the set target, or simply misses the target. The aim was to assess how many of these targets were successfully completed or not, and the rate at which they were completed or reached as per activity and per task, both horizontally and vertically.

11) Completion rate $(C R)$

As in the previous section, the CR of individual participants was assessed. The aim was to determine how fast participants were in terms of completing their tasks as individuals. The research team calculated the time it took to complete these tasks in that particular order, although they were not completed during the same session.

12) Time-based efficiency $(T B E)$

TBE is basically the assessment of one's computing efficiency per task and demonstrates the participant's adaptation and systems accommodation. It is measured by summing the CT of all participants per task and per activity and dividing it by the base time, as indicated in Table 1 .

13) Index of difficulty (ID)

The ability of a participant to master the usability of a system is based on the level of complexity of the system. Not all computing systems are the same. Some are more complex than others, some are more user friendly than others, and some are, by their interaction development, hard to master and use. Therefore, it was imperative to also pay attention to the GUI of all systems that were used as part of the system user assessment. Here Fitts' model comes back into play and the research team used it, as well as the system width and move distance, to determine the ID.

\section{4) Index of performance (IP)}

The system performance index, which distinguished all participants and set interactivity standards, is ultimately important regarding new and existing user-centric assessments. The IP and the ID are intertwined to establish overall system performance.

15) Interaction rate (IR)

IR is the HCI rate that is at the heart of this study. The research team calculated the user IR, which is the overall interaction for all actions, both vertically and horizontally, per task, activity, and position, as per Table 1.

16) $U X$

By calculating and assessing the UX, the ability of the user to master and control the system is demonstrated. For that reason, access would first need to be gained to the interaction (i), which is calculated by multiplying the system degree of aesthetics (e) and communication (ㄷ) , which are part of the system description. Access to the purpose (p) is also needed, which is established by adding the benefits (b) and the system status (s), which are known for various systems, and, finally, the speed ( $\underline{\mathrm{v}})$, which is obtained by summing the digital speed and the cognitive speed.

\section{RESEARCH QUESTION}

How can the implementation and usage of a model assist the evaluation of UX?

\section{RESEARCH METHODOLOGY}

This particular research is based on a quantitative research method. The use of mathematical formula here is vital. The identification of some critical variable above enables the researching team to collect data at the site where participants were asked to conduct an experiment, which involved the completion of a number of activities which were all filmed by the team with the consent of the participants, as shown in Figure 2. 
Participants were asked to complete a set of navigation activities using the provided low-fidelity prototype at first. The actual task consisted of reaching a particular section of the window at the request of the instructor and the time was then recorded. The second set of activities was based on reel hand-held computer systems, which were prepared for the occasion, in which instructor asked participants to reach a certain area of the interaction, e.g., compose an email. The instructor will then watch and record the action of the participant. Later the researcher then entered all the data collected into an Excel spreadsheet for analysis, using the formula shown below. The main goal was to test all the variable shown in Section 4

\section{FORMULA USED}

Having identified the areas and activities, participants were asked to complete a set of tasks from Phase 1 to Phase 5 , in accordance with the abovementioned activities that were designed to emulate the normal function of a computing system.

\section{1) Testing process and data collection}

Participants in this research project were people who had little or no contact with computing systems. The focus of this study is handheld computing systems. The overall experiment was conducted in three phases, with Phase 1 set to determine the state and readiness of the participants, measuring them on the same tasks but using various activities; then Phase 2, where we added more tasks; and lastly Phase 3, where we introduced other tasks.The aim of having more tasks andactivities is to avoid sticking to a single task which may routinely be mastered by participants to the extent of them completing them without the application of any form of cognition.

2) Data recording

During the experiment, we kept an eye on the time in

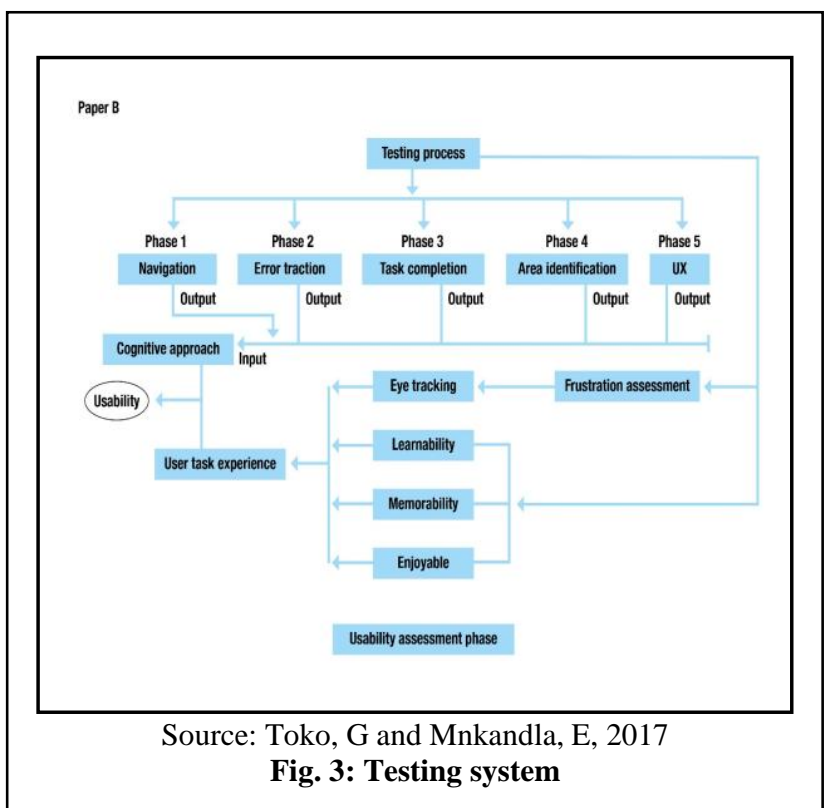

which tasks and activities were completed and recorded them in a Microsoft Excel spreadsheet, after which the data were analysed. In most cases, a video recorder was set up to capture the action of the participants, as well as their facial expressions.

\section{3) The role of benchmarking}

Effective and efficient assessment of UX can only have meaning if it can be compared with a known benchmark standard of system usability and, most importantly, its effect on the user. In other words, we are of the view that, in order to classify a user as having a low level of UX, there must be a comparison with a known and acceptable standard. Hence, we began this experiment by establishing a basic set of usability standards that are in line with what we were planning to measure the participants against. The benchmark standard was compiled prior to the interaction with participants and kept on the side for later comparison.

Figure 2 represents a single set of tasks. The research team asked participants to reach some point on the interface, for example, asking them to point or move from $\mathrm{A}$ to $\mathrm{B}$, or from $\mathrm{C}$ to $\mathrm{D}$. These actions are referred to as the calibration of the user's eye.

With the set of formulae (see Table 1) in place, we then developed an approach for testing. Figure 3 below shows how we conducted the testing.

From the onset, it is important to clearly state which tasks and activities contribute towards the improvement or affirmation of users when they are interacting with computing systems. Here, we have activities such as

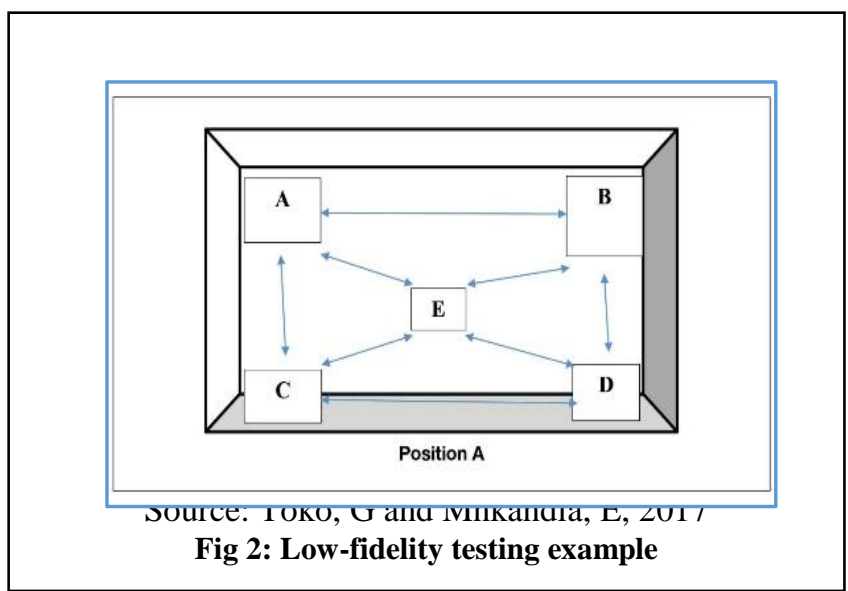

navigation, task completion, area identification, and error traction, which all lead to the overall UX.

The data we collected and entered into our system enabled us to visualise the performance of the participants. The participants were evaluated on two separate occasions, with the aim of identifying their level of computer-system knowledge before being selected to participate in the actual experiment. We therefore had two sets of data outputs: the data before and after the experiment.

4) How to read the table

Table 2 displays the data that were collected during the pre-training phase, with the aim of providing a better understanding of the participants before commencing. The research team gave the participants tasks that were similar to the actual tasks used in the experiment.

The table shows the 13 sets of instruments that were used to assess the participants, and, for each set, a few usability assessments were run and captured in the cell where they should be before moving to the next set.

The last column of the table shows the benchmarks against which the participants were measured. This shows whether a user was experienced or not. The purpose of this table is to indicate the initial level of computer usability. 
Table 1: Pre-experiment data

Pt. = Participant CNC $=$ Could Not Complete

Source: Toko, G and Mnkandla, E, 2017

\begin{tabular}{|c|c|c|c|c|c|c|c|c|c|c|}
\hline Instruments & Pt. 1 & Pt. 2 & Pt. 3 & Pt. 4 & Pt. 5 & Pt. 6 & Pt. 7 & Pt. 8 & Pt. 9 & Benchmark \\
\hline SR & $10 \%$ & $10 \%$ & $09 \%$ & $07 \%$ & $10 \%$ & $04 \%$ & $05 \%$ & $03 \%$ & $14 \%$ & $80-100 \%$ \\
\hline MT & $\begin{array}{l}12.1 \\
\text { min. }\end{array}$ & $\begin{array}{l}7.91 \\
\text { min. }\end{array}$ & $\begin{array}{l}11.2 \\
\text { min. }\end{array}$ & $\begin{array}{c}09.14 \\
\text { min. }\end{array}$ & $\begin{array}{l}10.3 \\
\text { min. }\end{array}$ & $\begin{array}{l}10.0 \\
\text { min. }\end{array}$ & $\begin{array}{l}08.1 \\
\text { min. }\end{array}$ & $\begin{array}{l}08.7 \\
\text { min. }\end{array}$ & $\begin{array}{l}08.5 \\
\text { min. }\end{array}$ & $0.005-0.030 \mathrm{~min}$. \\
\hline $\mathrm{AE}$ & $08 \%$ & $\mathrm{CNC}$ & $\mathrm{CNC}$ & $08 \%$ & $04 \%$ & $\mathrm{CNC}$ & $11 \%$ & $\mathrm{CNC}$ & $24 \%$ & $80-100 \%$ \\
\hline TS & $\begin{array}{l}10.2 \\
\mathrm{~cm} / \mathrm{s}\end{array}$ & $\begin{array}{l}11.3 \\
\mathrm{~cm} / \mathrm{s}\end{array}$ & $\begin{array}{c}0.9 \\
\mathrm{~cm} / \mathrm{s}\end{array}$ & $\begin{array}{l}12.2 \\
\mathrm{~cm} / \mathrm{s}\end{array}$ & $\begin{array}{l}13.1 \\
\mathrm{~cm} / \mathrm{s}\end{array}$ & $\begin{array}{l}15.2 \\
\mathrm{~cm} / \mathrm{s}\end{array}$ & $\begin{array}{l}16.2 \\
\mathrm{~cm} / \mathrm{s}\end{array}$ & $\begin{array}{l}13.1 \\
\mathrm{~cm} / \mathrm{s}\end{array}$ & $\begin{array}{l}11.0 \\
\mathrm{~cm} / \mathrm{s}\end{array}$ & $0.01-0.4 \mathrm{~s}$ \\
\hline US & $10 \%$ & $12 \%$ & $0.9 \%$ & $0.5 \%$ & $0.4 \%$ & $12 \%$ & $15 \%$ & $0.6 \%$ & $0.4 \%$ & $80-100 \%$ \\
\hline RT & $\mathrm{CNC}$ & $\mathrm{CNC}$ & $230 \mathrm{~s}$ & $254 \mathrm{~s}$ & $142 \mathrm{~s}$ & $\mathrm{CNC}$ & $210 s$ & $\mathrm{CNC}$ & $210 s$ & $9-12 \mathrm{~s}$ \\
\hline CRA & $08 \%$ & $06 \%$ & $\mathrm{CNC}$ & $12 \%$ & $14 \%$ & $\mathrm{CNC}$ & $09 \%$ & $\mathrm{CNC}$ & $13 \%$ & $80-100 \%$ \\
\hline CT & $900 \mathrm{~s}$ & $562 \mathrm{~s}$ & $561 \mathrm{~s}$ & $452 \mathrm{~s}$ & $352 \mathrm{~s}$ & $451 \mathrm{~s}$ & $540 \mathrm{~s}$ & $652 \mathrm{~s}$ & $532 \mathrm{~s}$ & $300-700 \mathrm{~s}$ \\
\hline TBE & $2.2 \mathrm{~s}$ & $\mathrm{CNC}$ & $5.9 \mathrm{~s}$ & $5.2 \mathrm{~s}$ & $7.14 \mathrm{~s}$ & $9.2 \mathrm{~s}$ & $5.2 \mathrm{~s}$ & $8.1 \mathrm{~s}$ & $\mathrm{CNC}$ & $4.2 \mathrm{~s}$ \\
\hline ID & 8.231 & 9.123 & 5.512 & 6.231 & 6.245 & 8.235 & 6.235 & 4.123 & 4.258 & 0.001-0.09 \\
\hline IP & 5.234 & 5.132 & 4.213 & 3.235 & 4.123 & 4.235 & 3.254 & 3.235 & 4.234 & $0.01-0.08$ \\
\hline DF & $90 \%$ & $88 \%$ & $91 \%$ & $95 \%$ & $96 \%$ & $88 \%$ & $85 \%$ & $94 \%$ & $96 \%$ & $0-10 \%$ \\
\hline AS & $\begin{array}{c}452 \\
\mathrm{~cm} / \mathrm{s}\end{array}$ & $\begin{array}{c}532 \\
\mathrm{~cm} / \mathrm{s}\end{array}$ & $\begin{array}{c}512 \\
\mathrm{~cm} / \mathrm{s}\end{array}$ & $\begin{array}{l}411 \\
\mathrm{~cm} / \mathrm{s}\end{array}$ & $\begin{array}{c}520 \\
\mathrm{~cm} / \mathrm{s}\end{array}$ & $\begin{array}{c}565 \\
\mathrm{~cm} / \mathrm{s}\end{array}$ & $\begin{array}{c}452 \\
\mathrm{~cm} / \mathrm{s}\end{array}$ & $\begin{array}{c}658 \\
\mathrm{~cm} / \mathrm{s}\end{array}$ & $\begin{array}{c}685 \\
\mathrm{~cm} / \mathrm{s}\end{array}$ & $0.001-0.97 \mathrm{~cm} / \mathrm{s}$ \\
\hline
\end{tabular}

Table 1: UX

Source: Toko, G and Mnkandla, E, 2017

\begin{tabular}{|c|c|c|c|c|c|c|c|c|c|c|}
\hline Instrument & $\begin{array}{c}\text { Bench } \\
\text { mark }\end{array}$ & Pt. 1 & Pt. 2 & Pt. 3 & Pt. 4 & Pt. 5 & Pt. 6 & Pt. 7 & Pt. 8 & Pt. 9 \\
\hline SR $(\%)$ & 90 & 35 & 75 & 42.5 & 65 & 77.5 & 62.5 & 57.5 & 80 & 62.5 \\
\hline $\mathrm{DF}(\%)$ & 25 & 65 & 25 & 57.5 & 35 & 22.5 & 37.5 & 42.5 & 20 & 37.5 \\
\hline ID (bits) & 0.001 & 0.468 & 0.585 & 0.585 & 0.585 & 0.585 & 0.585 & 0.468 & 0.585 & 0.585 \\
\hline IP (bits) & 0.005 & 0.005 & 0.009 & 0.005 & 0.008 & 0.009 & 0.01 & 0.007 & 0.008 & 0.01 \\
\hline TBE (goal/s) & 0.02 & 0.019 & 0.059 & 0.029 & 0.047 & 0.136 & 0.108 & 0.069 & 0.16 & 0.1 \\
\hline US (\%) & 90 & 65 & 25 & 57 & 35 & 22 & 37 & 42 & 20 & 37 \\
\hline $\mathrm{CT}(\mathrm{s})$ & 450 & 4079 & 917 & 1106 & 738 & 395 & 439 & 399 & 511 & 435 \\
\hline $\mathrm{RT}(\mathrm{s})$ & 11 & 2450 & 28 & 15 & 11 & 4 & 11 & 7 & 16 & 16 \\
\hline MT (s) & 500 & 1629 & 889 & 1091 & 727 & 391 & 428 & 392 & 495 & 419 \\
\hline $\mathrm{TS}(\mathrm{cm} / \mathrm{s})$ & 0.8 & 0.07 & 0.389 & 0.323 & 0.484 & 0.904 & 0.813 & 0.714 & 0.699 & 0.821 \\
\hline
\end{tabular}

Table 3: UX

Source: Toko, G and Mnkandla, E, 2017

\begin{tabular}{|c|c|c|c|c|c|c|c|c|c|c|}
\hline Instrument & $\begin{array}{c}\text { Benchm } \\
\text { ark }\end{array}$ & Pt. 1 & Pt. 2 & Pt. 3 & Pt. 4 & Pt. 5 & Pt. 6 & Pt. 7 & Pt. 8 & Pt. 9 \\
\hline SR $(\%)$ & 90 & 35 & 75 & 42.5 & 65 & 77.5 & 62.5 & 57.5 & 80 & 62.5 \\
\hline $\mathrm{DF}(\%)$ & 25 & 65 & 25 & 57.5 & 35 & 22.5 & 37.5 & 42.5 & 20 & 37.5 \\
\hline
\end{tabular}




\begin{tabular}{|c|c|c|c|c|c|c|c|c|c|c|}
\hline ID (bits) & 0.001 & 0.468 & 0.585 & 0.585 & 0.585 & 0.585 & 0.585 & 0.468 & 0.585 & 0.585 \\
\hline IP (bits) & 0.005 & 0.005 & 0.009 & 0.005 & 0.008 & 0.009 & 0.01 & 0.007 & 0.008 & 0.01 \\
\hline TBE (goal/s) & 0.02 & 0.019 & 0.059 & 0.029 & 0.047 & 0.136 & 0.108 & 0.069 & 0.16 & 0.1 \\
\hline US (\%) & 90 & 65 & 25 & 57 & 35 & 22 & 37 & 42 & 20 & 37 \\
\hline CT (s) & 450 & 4079 & 917 & 1106 & 738 & 395 & 439 & 399 & 511 & 435 \\
\hline RT (s) & 11 & 2450 & 28 & 15 & 11 & 4 & 11 & 7 & 16 & 16 \\
\hline MT (s) & 500 & 1629 & 889 & 1091 & 727 & 391 & 428 & 392 & 495 & 419 \\
\hline TS (cm/s) & 0.8 & 0.07 & 0.389 & 0.323 & 0.484 & 0.904 & 0.813 & 0.714 & 0.699 & 0.821 \\
\hline
\end{tabular}

The instruments are listed in the first column, while the participants are listed on the right.

\section{INTERPRETATION OF THE RESULTS}

Each participant in the experiment had five activities, which were all done in a series, and each activity had a series of tasks that had to be completed in chronological order. The pre-experimentation activities were known as Position A, B, and C, and the post-experiment activities were known as Position D, E, and F. In all cases, attention was paid to tasks within each activity to determine the overall performance of the participants, as well as the performance of all participants completing the same activities and tasks, whether horizontally or vertically.

Table 3 shows the ultimate output of this research, in other words, the UX metric that represents all the most important characteristics that are vital to determine potential UX. It gives a holistic and numeric approach, which is essential in comprehending tablet-system usability and adaptation. The UX metric is the assessment measurement that this current study attempts to address. It was developed based on a number of factors that are essential to comprehending mitigating variables that can assist user adaptation and depict future interaction.

1) Experiment findings

Concluding this segment requires that attention is paid to each instrument that was used and that their performances are re-evaluated.SR:

The SR of each participant improved after the training phase. Participant 4 had an SR of $100 \%$ for Activity A; thisis in line with the existing benchmark. When we compare this with the preliminary assessment, it appears that most participants improved from the poor performance initially observed.

MT: After the computation, it was evident that most participants improved. The benchmark here is between 0.005 and 0.030 minutes based on the activity completed, and the results show that the participants were well within the set standard.

AE: Based on the benchmark, an action is considered effective when the execution is between $80 \%$ and $100 \%$, which represents the number of tasks completed successfully divided by the number of tasks undertaken, and then multiplied by 100 to have the result as a percentage. Here we have a minimum of $42 \%$ and a maximum of $100 \%$. This may not be ideal but it generates a positive outlook.

TS: Based on the known benchmark, which is between 0.01 and 0.4 seconds, the calculation of the TS takes the distance travelled divided by the MT already calculated in the experiment. Most participants were still within the parameter, such as below 0.4 , but many were well above the limit, which is an area of concern.

US: According to our set benchmark, the US must be between $80 \%$ and $100 \%$. Here, there was an overall reading of $92 \%$, which is very much in line with the researchers' prediction and also indicates that some drastic measures were taken to improve the figure.

RT: The RT is determined by the idle time that is wasted between the time an instruction was given and the time the action was started. The benchmark indicates that it must be between 9 and 12 seconds to be acceptable; otherwise it can be viewed as wasted time. Here the participants' RT was within the limit; however, there were some isolated cases where it was outside the expected limit. The researchers ascribe these late reactions to external distractions such as system intimidation.

CRA: The CRA is the number of successful activities based on the number of activities available. The benchmark for this section is $80 \%$ to $100 \%$. While the average CRA for some participants was below the $65 \%$ mark, in most cases it was within the set bracket.

CT: The CT is the time it takes to complete the whole series of activities, and also the set of individual tasks that constitute that activity. By calculating the overall time it takes to complete all these, one may have an estimate of the speed of the participants' actions. The benchmark for this section is 300 to 900 seconds. Some participants took more time but in most cases these boundaries were met. In very few cases, the research team recorded times outside the upper limit of 900 seconds. TBE: Its benchmark is between 0.001 and 0.18 seconds. Here, the participants were well within the limit, as the lowest TBE was 0.01 seconds and the highest TBE was 0.17 seconds. However, there were isolated cases in which it was slightly over 1.7 seconds.

System ID: The calculation of the ID takes into account the distance travelled, the width of the target, as well as the log. It is important to note at this stage that both low- and highfidelity prototypes were the same sizes and therefore the width must be the same. This means that the output calculated for individual participants would be the same, and therefore we can use it as a mechanism to assess the validity of the outcomes. Here it stands at approximately 0.584 , which is within the set benchmark of 0.001 to 0.9 .

System IP: The calculation of this variable takes into account the ID, which is divided by the MT. The ID may be standard for all participants but the MT differs for each participant. The set benchmark is 0.01 to 0.08 and the results indicate that participants on average scored 0.004 . 
DF: The DF can be seen as the opposite of the task/activity SR. The set benchmark of $0 \%$ to $50 \%$ seems to be valid for any testing condition, or the lower the better in any condition. The figures here were a little high because some participants had a hard time completing the tasks. It therefore makes sense that the DF on average was approximately $44 \%$.

\section{VIII.CONCLUSION}

The ease of using a computing system is often driven by participants' understanding of the physical components of the device itself and their control thereof. Usability in most cases comes as a result of usage frequency, which itself emanates from the understanding of the goals that one seeks to achieve and UX therefore the summary of the above. By analysing Tables 2 and 3, one can easily identify where users fell short of the benchmark and where they seemed to excel. With this metric and proper understanding of user needs due to interactivity assessments, shortcomings can be remedied early enough during systems development. Tables 2 and 3 give us a clear measurement of the performance of individual participants in various tasks and activities, which at the end enabled us to compile the UX and for it to be useful to determine if the systems can be adopted by users.

\section{REFERENCES}

1. Adebajo, A. 2016. Thabo Mbeki. 1st edition. Johannesburg: Jacana Media.

2. Day, JS, Buchanan, G \& Makri, S. 2015. Learning lessons from controlled studies to investigate users' resilience strategies. Lecture Notes in Computer Science 9299:578-581.

3. Delanty, G. 2005. Social Science. UK: McGraw-Hill Education.

4. Duncan, G. 2015. Are table ergonomics a pain in the neck? Available at: www.digitaltrend.com/mobile/are-tablet-ergonomicsa-pain-in-the-neck/ (accessed on 20/01/ 2016).

5. Gilward, A, Moyo, M \& Stork, C. 2010. Comparative ICT sector performance review. Golbert 1(1):21-31.

6. Lee, C, Lee, J \& Kim, GJ. 2015. Touch skin: Proprioceptive input for small screen devices. Lecture Notes in Computer Science 9299:607-615.

7. Lee, M \& Kim, GJ. 2015. On applying sampling method to A/B testing of mobile applications: A case study. Lecture Notes in Computer Science 9297:203-214.

8. Leo de Oliveira, E, Miletto, EM \& Flores, LV. 2015. Redesigning interaction in CODES. Lecture Notes in Computer Science 9299:590-615.

9. Liang, H, Song, H, Fu, Y, Cai, X \& Zhang, Z. 2011. A remote usability testing platform for mobile phones, in Proceedings of the Computer Science and Automation Engineering (CSAE) 2011 IEEE International Conference. UK: Curran Associates: 312-316.

10. Marquardt, N. 2015. Sketching user experiences tutorial. Lecture Notes in Computer Science 9299:644-652.

11. McCarthy J \& Wright, P. 2010. The criticial potential of experience in experience-centred design. Available at: https://www.cl.cam.ac.uk/events/experiencingcriticaltheory/McCart hyWright-CriticalPotential.pdf (Accessed on 24/05/2017).

12. Ni, B, Al Mahmud, A \& Keyson, DV. 2015. Experiencing a home energy management system: Finding opportunities for design. Lecture Notes in Computer Science 9299:546-552.

13. Oz, E \& Jones, A. 2008. Management Information Systems. UK: Cengage Learning.

14. Prietch, SS \& Filgueiras, LVL. 2015. Technology acceptance evaluation by deaf students considering the inclusive education context. Lecture Notes in Computer Science 9296:20-37.

15. Reiss, E. 2012. Usable Usability: Simple Steps for Making Stuff Better. USA: Wiley.

16. Shely, GB, Cashman, TJ \& Walker, TJ. 2015. Discovering Computers. 10th edition. USA: Course Technology.

17. Toko, G \& Mnkandla, E. 2011. Open source system: Migration and adoption for education purposes in a developing nation, in Proceedings of the Second International Conference on Computer Science Education: Innovation and Technology. USA: ACM:22512195.
18. Toko, G \& Mnkandla, E. 2017. Closing the digital gap: handheld computing, adult first-time users, and a user experience metric model, submitted as part of a PHD qualification, university of south africa.

19. Turban, E, Sharda, R, Delen, D \& King, D. 2008. Business Intelligence. 2nd edition. USA: Prentice Hall.

20. Vatavu, RD, Anthony, L \& Brown, Q. 2015. Child or adult? Inferring smartphone users' age group from touch measurements alone. Lecture Notes in Computer Science 9299:1-9.Yu, J \& Kim, G. 2015. Eye strain from switching focus in optical see-through displays. Lecture Notes in Computer Science 9299:550-56 